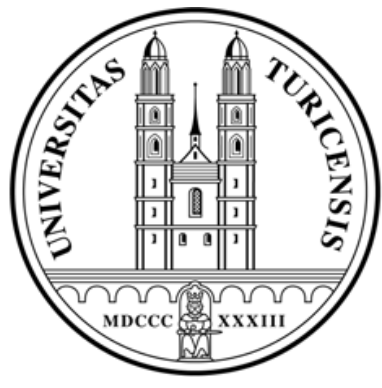

Institute for Empirical Research in Economics

University of Zurich

Working Paper Series

ISSN 1424-0459

Working Paper No. 189

Signaling, Globality, and the Intuitive Criterion

Christian Ewerhart and Philipp Wichardt

May 2004 


\title{
Signaling, Globality, and the Intuitive Criterion*
}

\author{
Christian Ewerhart ${ }^{\dagger}$ \\ Institute for Empirical Research in Economics (IEW) \\ University of Zurich \\ Philipp Wichardt ${ }^{\ddagger}$ \\ Department of Economics \\ University of Bonn
}

May 2004

\footnotetext{
*Acknowledgements: Ewerhart would like to thank Hans Carlsson for an extensive discussion about the theory of global games. Wichardt thanks the European Commission for financial support and the University College London for its hospitality.

${ }^{\dagger}$ E-mail: christian.ewerhart@iew.unizh.ch.

${ }^{\ddagger}$ Postal address for correspondence: Bonn Graduate School of Economics, Adenauerallee 24-26, D-53113 Bonn, Germany; e-mail: p.c.w@web.de.
} 


\begin{abstract}
A global signaling game is a sender-receiver game in which the sender is only imperfectly informed about the receiver's preferences. The paper considers an economically relevant class of signaling games that possess more than one Perfect Bayesian equilibrium. For this class of games, it is shown that a Perfect Bayesian equilibrium is unaffected by a small perturbation of the information structure if and only if it is consistent with a criterion suggested by Cho and Kreps (1987). Moreover, the equilibrium in the global signaling game is essentially unique.
\end{abstract}

Key words: Global Games, Signaling, Intuitive Criterion JEL code: C72 


\section{Introduction}

The economic notion of signaling, as introduced by Spence (1973), refers to a specific situation of asymmetric information in which an informed and firstmoving agent, the sender, seeks to influence the subsequent decision of an a priori uninformed observer, the receiver. The literature has worked so far under the assumption that the preferences of the receiver are common knowledge. However, this assumption may not be satisfied under all circumstances. E.g. in the example of educational signaling, it is hard to imagine that future wage levels can be predicted with absolute certainty. For another example, consider a firm that pays dividends to stock holders to signal future profitability. Such a firm will not always be certain about the marginal tax rate of the average equity owner. Finally, and closer to the current framework, an incumbent monopolist adhering to limit pricing will typically only be imperfectly informed about the profitability of market entry for an outsider. It should be clear that this list of examples could be continued without much difficulty. Thus, there appear to exist some settings of interest in which the sender of an economically relevant signal is uncertain about the preferences of the party that receives the signal.

From a methodological point of view, the introduction of incomplete information has proven to be of significant value. Carlsson and van Damme (1993) have shown that in two-by-two coordination games, a unique prediction of the risk dominant equilibrium is feasible when the game is embedded into a global payoff manifold, and when the information structure is only slightly perturbed. The underlying theory has recently created a number of interesting economic applications. Morris and Shin (1998), for example, show that the introduction of incomplete information yields a unique prediction concerning the outcome of a coordination problem between a continuum of foreign exchange traders. This allows to derive unprecedented comparative statics results, e.g. analyzing the consequences of a Tobin tax on currency speculation. This and other recent applications of global games, as surveyed by Morris and Shin (2003), have in common that the number of equilibria in a given game is significantly reduced by eliminating the coordination problem between a population of agents that make their decision virtually at the same moment of time.

Also the notorious multiplicity of equilibria in signaling games, while not always considered from this perspective, is due to a coordination problem between players that move at the same stage. Early studies in this field resolved the coordination problem by imposing "plausible" beliefs at information sets that remain unreached in equilibrium. Cho and Kreps (1987), for 
example, have suggested the Intuitive Criterion requiring that the receiver in a signaling game, after observing an unexpected deviation from equilibrium play, should assign probability zero to all those sender types that, by deviating like that, cannot improve their respective payoff, provided the receiver makes some utility-maximizing choice of response. However, this and similar concepts, while a formal consequence of Kohlberg and Mertens' (1986) strategic stability, have been criticised for not being derivable from more general principles of belief formation in extensive-form games. ${ }^{1}$

Given this background, it appeared to us as not unreasonable to apply the global games methodology to signaling games. In fact, when globality is interpreted in a sufficiently broad sense, then we are not the very first to do so. Carlsson and Dasgupta (1997) have considered informational robustness of equilibria in signaling games. In contrast to this paper, however, Carlsson and Dasgupta study informational perturbation in which the receiver obtains an imperfect signal of the sender's message. This assumption turns out to have a considerable power: It is shown that the set of noise-proof equilibria is strictly contained in the set of equilibria that survive the never-a-weakbest-reply criterion.

In this paper, we study the stability of signaling equilibria with respect to informational perturbations concerning the receiver's preferences. This means considering games of two-sided incomplete information where in addition to the usual uncertainty about the sender's type, there is incomplete information about the receiver's preferences. We consider a robust class of simple signaling games based on the well known beer-quiche game (Cho and Kreps, 1987) with two sender types, two possible responses, and a continuum of receiver types. For this class of games, we find that a Perfect Bayesian Equilibrium is informationally stable in the suggested way if and only if it is consistent with the Intuitive Criterion.

The easier part of this result is to show that an informationally stable Perfect Bayesian Equilibrium must be consistent with the Criterion. In fact, it turns out that this direction can be shown using the established methodologies from the theory of global games. Specifically, the argument begins with the insight that the receiver possesses a dominant strategy when her preferences are sufficiently extreme. The desired result then follows from iterated dominance eliminations. The second part of the claim, vz. that any equilibrium consistent with the Criterion is informationally stable, requires

\footnotetext{
${ }^{1}$ This critique is at least partly refuted by Battigalli and Siniscalchi (2002), who formalize epistemic conditions for the Intuitive Criterion.
} 
to find a Perfect Bayesian Equilibrium in the global game. This task turns out to be somewhat more intricate. The key step in the proof is to relate the set of equilibria in the global signaling game to the set of equilibria in a global two-by-two (non-coordination) normal-form game. A general result by Milgrom and Weber (1985) then guarantees existence of the equilibrium in the static game and closes the argument.

The rest of the paper is structured as follows. In Section 2, we discuss an example neglecting technicalities for the purpose of accessibility. In Section 3 , the general setup is introduced. Section 3 also contains the statement and proof of our first main result, that links informational stability to the Intuitive Criterion. Section 4 discusses uniqueness properties. In Section 5, we prove existence of the equilibrium. In Section 6 we solely focus on the example from Section 2 and explicitly construct an equilibrium for a certain range of information structures. Section 7 concludes. Technical steps of the proofs are gathered in the appendix.

\section{An example}

In this section, we will illustrate our main results using a global version of a game introduced by Cho and Kreps (1987). See Figure 1 for a graphical representation of the game tree.

There are two players, Player 1 (the sender) and Player 2 (the receiver). The game has four stages. In the initial stage of the game, Nature determines both the type $t \in T$ of Player 2, and the type of Player 1, composed of a preference parameter $\theta \in\{\underline{\theta}, \bar{\theta}\}$ and an imperfect signal $\tilde{t}$ about Player 2's type. We will assume for concreteness that the probability of $\bar{\theta}$ is given by $p=0.9$, and that the conditional distribution of $\tilde{t}$ is uniform on $[t-\varepsilon, t+\varepsilon]$, where $\varepsilon>0$. In the second stage, Player 1 observes $\theta$ and $\tilde{t}$ and chooses a message $m \in\{\underline{m}, \bar{m}\}$. In the third stage, Player 2 observes her type $t$ and the message $m$, but neither the sender's type $\theta$ nor the imperfect signal $\tilde{t}$, and chooses a response $r \in\{\underline{r}, \bar{r}\}$. Finally, payoffs are determined according to utility functions $u_{1}(m, r, \theta)$ and $u_{2}(r, \theta, t)$ as shown in Figure 1.

As can be seen from this specification, the global signaling game differs from a signaling game in that the preferences of the receiver are only imperfectly observable for the sender.

We denote the global games version of the signaling game by $G(\varepsilon)$. The error parameter $\varepsilon$ captures the degree to which the sender possesses incomplete 
information about the receiver. In the limit case, i.e. for $\varepsilon=0$, the sender is perfectly informed about the receiver's type $t$. Thus, once $t$ has been determined by nature, a signaling game, referred to as $G_{t}(0)$, is played. We will show that for $\varepsilon$ sufficiently small, and for $\tilde{t}$ close to 0 , the sender chooses the message $\bar{m}$ with probability one for both possible values of $\theta$. Notice that this outcome, i.e. pooling on $\bar{m}$, is predicted in the local game $G_{0}(0)$ by the Intuitive Criterion.

We begin the analysis of the example by showing that, given our setting, Player $1 \bar{\theta}$ (the high type of Player 1 ) will choose $\bar{m}$ for a wide range of signals $\tilde{t}$. This finding is independent of $\varepsilon$ and, thus, already upsets the $\underline{m}$ pooling equilibrium. The initial dominance argument is to note that for very low values of the preference parameter $t$, Player 2 has a dominant strategy. What matters for the argument is the response to message $\bar{m}$.

Fact 1 . For $t<-1$, Player $2 \bar{m}$ chooses $\underline{r}$.

The next step is to analyze the decision of Player 1 when confronted with a very low signal. For this, assume that it is already shown for some $t^{*}$ that for any $t<t^{*}$, Player $2 \bar{m}$ responds with $\underline{r}$. This assumption has been verified for $t^{*}=-1$ so far. Consider now a signal $\tilde{t}<t^{*}$ for Player $1 \bar{\theta}$. Because noise is symmetric, Player 1's posterior then entails that Player $2 \bar{m}$ will choose $\bar{r}$ with probability less than $1 / 2$. In this case, however, the message $\bar{m}$ promises a payoff to Player $1 \bar{\theta}$ that is strictly larger than 2 , so that, in expected terms, $\bar{m}$ is strictly better than $\underline{m}$ for Player $1 \bar{\theta}$. We summarize the argument as follows:

Fact 2. Assume that for all $t<t^{*}$, Player $2 \bar{m}$ chooses $\underline{r}$. Then for any $\tilde{t}<t^{*}$, Player $1 \bar{\theta}$ will choose $\bar{m}$.

Consider now the posterior of Player $2 \bar{m}$. Assume that Player $1 \bar{\theta}$ plays $\bar{m}$ for all values $\tilde{t}<t^{*}$, for some fixed value $t^{*}$. Let Player $2 \bar{m}$ be of type $t<t^{*}+\varepsilon$. Given such $t$, Player 2 knows that the probability of Player 1 receiving a signal $\tilde{t}<t^{*}$ is strictly positive. By assumption, Player 1 chooses $\bar{m}$ for all values $\tilde{t}<t^{*}$. Thus, Player $2 \bar{m}$ assigns some strictly positive probability to $\theta=\bar{\theta}$.

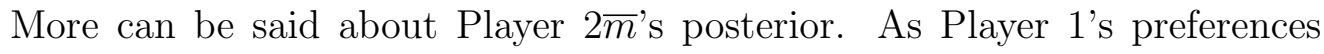
exhibit increasing differences with respect to $\theta$ and $m$, Player 2 can infer that whenever $1 \underline{\theta}$ has a weak preference for $\bar{m}, 1 \bar{\theta}$ has a strict preference for $\bar{m}$, given the signal $\tilde{t}^{2}$ Hence, the posterior of facing a high type $\bar{\theta}$ on

\footnotetext{
${ }^{2}$ If $p^{\prime}$ is the posterior probability of $1 \underline{\theta}$ playing $\bar{m}$, then the posterior probability of $1 \bar{\theta}$ playing $\bar{m}$ must be at least $p^{\prime}$.
} 
message $\bar{m}$ cannot fall below the prior probability $p$ of the high type. If we denote the belief of Player 2 about the possible sender of a message $m$ given her type $t$ by $\mu(. \mid m, t)$, this can be expressed as:

$$
\mu(\bar{\theta} \mid \bar{m}, t) \geq p=0.9 \text {. }
$$

The expected payoff for Player $2 \bar{m}$ from $\bar{r}$, denoted by $\pi_{2}(\bar{r} \mid \bar{m}, t)$, is therefore bounded from above by

$$
\pi_{2}(\bar{r} \mid \bar{m}, t) \leq(1+t) \cdot 0.1+(t-1) \cdot 0.9 .
$$

However, even this upper bound falls below the zero payoff from $\underline{r}$ whenever $t<0.8$. So we have shown:

Fact 3. Assume that $1 \bar{\theta}$ plays $\bar{m}$ for all values $\tilde{t}<t^{*}<0.8$. Then $2 \bar{m}$ chooses $\underline{r}$ for all $t<t^{*}$.

Putting the above facts together delivers the result that for any signal $\tilde{t}<0.8$, Player $1 \bar{\theta}$ chooses $\bar{m}$. This proves that pooling on $\underline{m}$ is not an informationally stable equilibrium of the signaling game $G_{0}(0)$. Note that up to this point only dominance arguments have been applied.

One can go a bit further and show that any equilibrium in the global game involves pooling on $\bar{m}$ for values $\tilde{t}<0.8$. To provoke a contradiction, assume an equilibrium in which $1 \underline{\theta}$ plays $\underline{m}$ with positive probability in an $\varepsilon / 4$ neighbourhood $U$ of a given $\tilde{t}<0.8-2 \varepsilon$. See Figure 2 for illustration. Because the $\bar{\theta}$ type does not choose $\underline{m}$ in the considered area, Player $2 \underline{m}$ assigns probability one to the $\underline{\theta}$ type whenever

$$
t \in\left(\tilde{t}-\frac{3 \varepsilon}{4} ; \tilde{t}+\frac{3 \varepsilon}{4}\right) .
$$

Thus, for all $t$ satisfying (1), Player $2 m$ certainly responds with $\bar{r}$. If we now consider the expectation of Player $1 \underline{\theta}$ given $\tilde{t} \in U$, we find that she has to assign a probability larger than $3 / 4$ to a response $\bar{r}$ against message $\underline{m}$. Hence, Player $1 \underline{\theta}$ would strictly prefer to choose $\bar{m}$ for all $\tilde{t} \in U$. This yields the desired contradiction, and we have shown:

Fact 4. In any equilibrium of the global game, Player $1 \underline{\theta}$ must choose $\bar{m}$ for all signals $\tilde{t}<0.8-2 \varepsilon$ (and player $1 \bar{\theta}$ must choose $\bar{m} \overline{\text { for all }} \tilde{t}<0.8$ ).

Thus, for $t=0$, only pooling on $\bar{m}$ followed by $\underline{r}$ can be an equilibrium in the global signaling game. We will proceed with the analysis of this example 
in Section 4 , where we show that pooling on $\bar{m}$ is indeed an informationally stable equilibrium.

\section{The general framework}

A global signaling game involves two parties, the sender (Player 1), and the receiver (Player 2). As mentioned before, the modification vis-à-vis the text-book signaling game is that the sender has only incomplete information about the utility function of the receiver. Due to the introduction of a further move by nature to determine $t$ and $\tilde{t}$, a more complex information structure arises and the usual notion of types becomes somewhat awkward. Strictly speaking, a type of Player 1 is a combination $(\theta, \tilde{t})$. However, for the sake of convenience, we will refer to $\theta$ as Player 1's global type. Moreover, we will talk about Player $1 \theta$ referring to the global agent $\theta$ of Player 1, i.e. Player 1 at her global information set $\theta$. Similarly, we will talk about Player $2 m$ referring to Player 2 at her global information set $m$. We will call pairs $(\theta, \tilde{t})$ and $(m, t)$ local information sets of Player 1 and Player 2, respectively. ${ }^{3}$ We denote the sets of possible global types and signals for Player 1 by $\Theta$ and $\widetilde{T}$, and the set of possible types of Player 2 by $T$. The set of possible messages for Player 1 is denoted by $M$ and the set of possible responses for Player 2 by $R$. The only restriction made at this point is that $\Theta, M$ and $R$ are all finite, and that $T, \widetilde{T} \subseteq \mathbb{R}$.

The resulting global signaling game, again denoted by $G(\varepsilon)$, has four stages. In the first stage, nature determines a global type and a signal $(\theta, \tilde{t}) \in \Theta \times$ $\widetilde{T}$ for Player 1 , and a type $t \in T$ for Player 2 . We will assume that the pair $(t, \tilde{t})$ and the individual parameter $\theta$ are drawn independently, but no such restrictions will be imposed on the joint distribution of $t$ and $\tilde{t}^{4}$ The probability distribution over $\Theta$ will be denoted by $p($.$) , the joint distribution$ of $(t, \tilde{t})$ by $\nu$. We assume that $\nu$ and its marginal distributions are atomless. The corresponding conditional densities for $\tilde{t}$ and $t$ are assumed to exist, and denoted by $\nu(. \mid t)$ and $\nu(. \mid \tilde{t})$, respectively. In the second stage, Player 1 observes her global type and her signal and sends a message $m(\theta, \tilde{t}) \in M$. In stage 3 , the receiver observes her own type $t$ and the sender's message, but neither her type nor her signal, and chooses a response $r(m, t) \in R$. Finally, utility is determined by $u_{1}(m, r, \theta)$ for the sender, and by $u_{2}(r, \theta, t)$ for the receiver.

\footnotetext{
${ }^{3}$ Taking a slightly different perspective $(\theta, \tilde{t})$ can also be viewed as a local type or agent of Player 1.

${ }^{4}$ In fact, if $t$ and $\tilde{t}$ are independent, then the sender's signal is without value, so that we have a signaling game with uncertain payoff for the receiver.
} 
It turns out that, in general, existence of an equilibrium in pure strategies is not obvious (see Section 5), so that some notion of mixed strategy is desirable. However, as pointed out by Aumann (1964), the choice of a model for mixing is not straightforward in the context of Bayesian games with large type spaces. Fortunately, for our situation there is the very intuitive approach suggested by Milgrom and Weber (1985). Specifically, a distributional strategy for Player $1 \theta, \theta \in \Theta$, is a probability distribution $\psi_{1 \theta}$ on $\widetilde{T} \times M$ such that the marginal distribution on $\widetilde{T}$ induced by $\psi_{1 \theta}$ is identical to the marginal distribution of $\tilde{t}$ on $\widetilde{T}$. A distributional strategy for Player $2 m, m \in M$, is a probability distribution $\psi_{2 m}$ on $T \times R$ such that the marginal distribution on $T$ induced by $\psi_{2 m}$ is identical to the marginal distribution of $t$ on $T$.

Lemma 1. Without loss of generality, the distributional strategies of sender and receiver can be represented by measurable functions

$$
\begin{gathered}
\sigma_{1}: \Theta \times \widetilde{T} \rightarrow \Delta(M), \\
\sigma_{2}: M \times T \rightarrow \Delta(R),
\end{gathered}
$$

where $\Delta(X)$ denotes the set of probability distributions over the (finite) set $X$.

Proof. Consider the sender first. According to the assumptions made, the marginal distribution of $\tilde{t}$ is atomless. Therefore, the cumulative distribution function (cdf) of $\tilde{t}$ cannot possess any jumps. As a cdf of $\tilde{t}$ is the weighted sum of the conditional cdfs for the corresponding finitely many messages in $M$ and since cdfs are nondecreasing, the cdfs of the messages cannot possess jumps either. Hence, the cdfs for the messages are atomless and, therefore, the corresponding distributions must possess densities. A similar argument applies to the receiver.

The equilibrium notion that will be employed is that of a Perfect Bayesian Equilibrium (Fudenberg and Tirole, 1991a). A pure strategy for the sender is a message $m(\theta, \tilde{t})$ conditional on the sender's global type, and on the signal received about the receiver's type. The corresponding mixed strategy is denoted by $\sigma_{1}(\theta, \tilde{t})$. Similarly, a pure strategy for the receiver is an action $r(m, t)$ conditional on the message received, and conditional on the receiver's type. The corresponding mixed strategy is denoted by $\sigma_{2}(m, t)$. By abuse of notation, the probability weights on the respective actions are denoted by $\sigma_{1}(m, \theta, \tilde{t})$ and $\sigma_{2}(r, m, t)$.

At each information set, the receiver will form beliefs about the sender's global type. For all pairs $(m, t)$, these beliefs are given by a function

$$
\mu(. \mid m, t): \Theta \longrightarrow[0,1] .
$$


We now can state the formal definition of a perfect Bayesian Equilibrium.

Definition 1. A perfect Bayesian equilibrium (PBE) of a global signaling game consists of a strategy profile $\sigma^{*}=\left(\sigma_{1}^{*}, \sigma_{2}^{*}\right)$ and beliefs $\mu(. \mid m, t)$ such that

1. for all $\left(m^{*}, \theta, \tilde{t}\right)$, if $\sigma_{1}^{*}\left(m^{*}, \theta, \tilde{t}\right)>0$ then

$$
m^{*} \in \arg \max _{m} \sum_{r} u_{1}(m, r, \theta) \operatorname{Pr}(r \mid m ; \tilde{t}),
$$

where

$$
\operatorname{Pr}(r \mid m ; \tilde{t}):=\int \sigma_{2}^{*}(r, m, t) d \nu(t \mid \tilde{t})
$$

2. for all $\left(r^{*}, m, t\right)$, if $\sigma_{2}^{*}\left(r^{*}, m, t\right)>0$, then

$$
r^{*} \in \arg \max _{r} \pi_{2}(r \mid m, t)
$$

where

$$
\pi_{2}(r \mid m, t):=\sum_{\theta} u_{2}(r, \theta, t) \mu(\theta \mid m, t)
$$

3. for all $(\theta, m, t)$, if the information set of the receiver corresponding to $m$ and $t$ is reached in equilibrium with strictly positive probability, i.e., if

$$
\sum_{\theta^{\prime}} p\left(\theta^{\prime}\right) \cdot \int \sigma_{1}^{*}\left(m, \theta^{\prime}, \tilde{t}\right) d \nu(\tilde{t} \mid t)>0
$$

then

$$
\mu(\theta \mid m, t)=\frac{p(\theta) \cdot \int \sigma_{1}^{*}(m, \theta, \tilde{t}) d \nu(\tilde{t} \mid t)}{\sum_{\theta^{\prime}} p\left(\theta^{\prime}\right) \cdot \int \sigma_{1}^{*}\left(m, \theta^{\prime}, \tilde{t}\right) d \nu(\tilde{t} \mid t)} .
$$

In this explorative study, we confine our attention to the following class of signaling games based on the earlier example with $M=\{\underline{m}, \bar{m}\}$ and $R=\{\underline{r}, \bar{r}\}$. We replace the numeric payoffs by parameters as shown in Figure 3, requiring that:

$$
a, b, c, d, x, y>0 .
$$

Furthermore, we assume, that the possible types $t$ of Player 2 are uniformly distributed over an interval $T:=[-K, K]$ where $K \gg 0$. Player 1's signal $\tilde{t}$ here is assumed to be uniform on $[t-\varepsilon, t+\varepsilon]$, in particular $\widetilde{T}=[-K-\varepsilon, K+\varepsilon]$. 
This implies that the posterior on $t$ given $\tilde{t}$ is also uniform on $[t-\varepsilon, t+\varepsilon]$ if $\tilde{t} \in[-K+\varepsilon, K-\varepsilon] .{ }^{5}$ Moreover, we require that $K$ is sufficiently large not to allow boundary effects to interfere with the argument, i.e. $K \gg \max \{x, y\}$.

We make one additional assumption:

$$
c>a \text { and } b>d \text { and } p>\frac{x}{x+y}
$$

Assumption A ensures that both $(\bar{m}, \bar{m})$ and $(\underline{m}, \underline{m})$ are Perfect Bayesian Equilibria in $G_{0}(0)$, the unperturbed game with $t=0$. Moreover, it is not difficult to check that under Assumption A, the equilibrium $(\underline{m}, \underline{m})$ is always eliminated by the Intuitive Criterion, whereas $(\bar{m}, \bar{m})$ is not. The proof of the next result follows closely the discussion of the example in Section 2.

Theorem 1. In any PBE, of the global sender types pool on $\bar{m}$ in response to a signal $\tilde{t}$ close to zero.

Proof. See the appendix.

The theorem shows that pooling on $\underline{m}$ cannot be an informationally stable equilibrium of the underlying signaling game $G_{0}(0)$. However, note that the theorem leaves open the question of existence. Without existence of a PBE in the global game, a selection theory as suggested by Theorem 1 may have no bite. This issue will be addressed in Section 5. It is convenient to discuss uniqueness properties of the global equilibrium first.

\section{Uniqueness}

In Section 3 it was shown that, in any equilibrium of the global signaling game, both global types of sender pool on $\bar{m}$ in a neighbourhood of $\tilde{t}=0$. In this section, we show that, apart from a small area of types $t$ and signals $\tilde{t}$ in which the transition between $\bar{m}$ and $\underline{m}$ of Player $1 \underline{\theta^{\prime}} s$ strategy takes place, equilibrium strategies are unique up to beliefs off the equilibrium path. We will refer to the remaining transition-area as "black hole." In Section 6, when we construct a concrete equilibrium for our example game from Section 2, we will show that even within the black hole there is only little latitude for strategy choices.

We will now give the arguments needed to sustain uniqueness of strategies on the equilibrium path but outside the black hole for our example discussed

\footnotetext{
${ }^{5}$ For the argument to work, it suffices to assume that $\tilde{t}=t+\delta$, where $\delta$ is determined according to some distribution on $[-\varepsilon,+\varepsilon]$ that possess a strictly positive density.
} 
in Section 2. The general proof is slightly more technical and can be found in the appendix.

In order to see that there is not much freedom of choice in the example game if equilibrium is to be reached, we complement the results from Section 2 using the obvious dominance arguments. Doing this, we obtain that in any equilibrium of the global game $G(\varepsilon)$

- $1 \bar{\theta}$ has to choose $\bar{m}$ for all values $\tilde{t}<0.8$ and $\tilde{t}>1$;

- $1 \underline{\theta}$ has to choose $\underline{m}$ for $\tilde{t} \leq-1$ and $\tilde{t}>1$, and message $\bar{m}$ for $\tilde{t} \in$ $(-1,0.8-2 \varepsilon)$

- $2 \bar{m}$ has to choose $\underline{r}$ for $t<0.8$, and $\bar{r}$ for $t>1$;

- $2 \underline{m}$ has to choose $\underline{r}$ for $t<-1$ and $\bar{r}$ for $t>1$.

The next step is to note that $G(\varepsilon)$ exhibits a single crossing property. Specifically, whatever the strategy of Player 2 , a weak preference of Player $1 \bar{\theta}$ for $\underline{m}$ implies a strict preference of Player $1 \underline{\theta}$ for message $\underline{m}$. Hence, if in equilibrium

$$
\mu(\bar{\theta} \mid \underline{m}, t)>0 \text {, then we must have } \mu(\bar{\theta} \mid \underline{m}, t) \leq p .
$$

As we have seen in Section 2, for $t \geq 0.8$, it is optimal for Player 2 to respond with $\bar{r}$ if the proportion of low global types expected is at least $1-p$. Thus, Player 2 will play $\bar{r}$ against $\underline{m}$ for all types $t>0.8$, whenever her information set is reached. An immediate consequence of this is that Player $1 \bar{\theta}$ will never play $\underline{m}$. This is because for all types $t$ of Player 2 for which Player $1 \bar{\theta}$ cannot be sure that Player $2 \bar{m}$ will play $\underline{r}$ she can be sure that Player $2 \underline{m}$ will play $\bar{r}$.

Bearing this in mind, we now are able to extend the argument given given in Section 2 to support $\bar{m}$ for Player $1 \underline{\theta}$ given signals $\tilde{t} \in(-1,0.8-2 \varepsilon)$ up to values $\tilde{t}<0.8$. Loosely speaking, since Player $2 \underline{m}$, given $t>-1$, would always fight if her information set was reached, and since Player 1, for all signals $\tilde{t} \in[0.8-2 \varepsilon, 0.8)$, will believe that

$$
\operatorname{Pr}(\underline{r} \mid \bar{m} ; \tilde{t})>0.5
$$

it is optimal for Player $1 \underline{\theta}$ to opt for $\bar{m}$ also for signals $\tilde{t} \in[0.8-2 \varepsilon, 0.8){ }^{6}$ Thus, we have shown that in any equilibrium of $G(\varepsilon)$ we must have that:

\footnotetext{
${ }^{6}$ More formally, we would have to argue again in terms of measurable fractions of Player $1 \underline{\theta}$ since single types, being of measure 0 , would not affect the beliefs of Player $2 \underline{m}$. The argument then follows similar lines as the one given to establish Fact 4 in Section 2 and is left out at this place.
} 
Player $1 \bar{\theta}$ plays $\bar{m}$ irrespective of her signal; Player $1 \underline{\theta}$ plays $\underline{m}$ for $\tilde{t} \leq-1$ and $\tilde{t}>1$, and message $\bar{m}$ for $\tilde{t} \in(-1,0.8)$; Player $2 \bar{m}$ plays $\underline{r}$ for $t<0.8$, and $\bar{r}$ for $t>1$; and Player $2 \underline{m}$ plays $\underline{r}$ for $t<-1$ and $\bar{r}$ for $t>1$ and for all types $t \in[-1,0.8]$ plays such that Player $1 \underline{\theta}$, given a signal $\tilde{t} \in(-1,0.8)$, is deterred from sending message $\underline{m}$.

The results obtained so far uniquely determine the behavior of players for types $t$ and signals $\tilde{t}$ outside of the interval $(0.8,1)$, allowing deviations only on sets of measure zero. Theorem 2 summarises the results in more general terms. See Figure 4 for illustration; notice that in case of the example game we have $x^{\circ}=x=1, y^{\circ}=y=1$ and $t^{\circ}=t^{*}=0.8$.

Theorem 2. Take any equilibrium and consider the path, i.e. the distribu- 4 tion over end nodes, of this equilibrium. The conditional distribution, outside here the black hole, is unique.

Proof. See text above and the appendix for the general case.

The Theorem shows that, while uniqueness of the equilibrium in the global game cannot be satisfied, the differences of any two equilibria are restricted to the area of bilateral mixing. In this area, as we have seen, uncertainty about $t$ and $\tilde{t}$ and the necessity of only perceived mixing (i.e. on average over an interval) allows for some freedom of choice.

\section{Existence}

In a global signaling game, type spaces are continuous, and typically not all local information sets are reached in equilibrium. ${ }^{7}$ As a consequence, existence of an equilibrium is not completely straightforward. The method of proof that we choose is to make an "educated guess" concerning the equilibrium strategies for a wide range of type-signal pairs. In fact, as we have seen above, this educated guess is essentially a necessary consequence of equilibrium conditions. For values of $t$ and $\tilde{t}$ in the remaining black hole area, actual strategies are difficult to pin down (see also Figure 4). However, it turns out that the remaining game can be reduced to a static Bayesian game for which existence results are available.

\footnotetext{
${ }^{7}$ Note that according to Definition 1 , a local information set $(m, t)$ of player 2 is reached in equilibrium if the conditional probability that the message $m$ is chosen in equilibrium, conditional on $|\tilde{t}-t|<\varepsilon$, is strictly positive.
} 
In the following, we show how the needed argument works for the example game presented in Section 2. We again confine our discussion to this particular example, since the technical detail of the general analysis do not add much to the argument but are likely to detract the readers attention form the main points. The technicalities can be found in the appendix.

To move on, consider the following incomplete candidate for an equilibrium strategy based on the results from the previous section (cf. Figure 4):

- Player $1 \bar{\theta}$ chooses $\bar{m}$ throughout.

- Player $1 \underline{\theta}$ chooses message $\underline{m}$ for $\tilde{t} \leq-1$, message $\bar{m}$ for $\tilde{t} \in(-1,0.8)$, and message $\underline{m}$ for $\tilde{t}>1$.

- Player $2 \bar{m}$ chooses $\underline{r}$ for $t<0.8$, and $\bar{r}$ for $t>1$.

- Player $2 \underline{m}$ chooses $\underline{r}$ for $t<-1$, and $\bar{r}$ for $t \geq-1$.

This specification fully describes choices of actions for Players $1 \bar{\theta}$ and $2 \underline{m}$. Moreover, it describes choices of action for Players $1 \underline{\theta}$ and $2 \bar{m}$ outside of the interval $[0.8,1]$. For the sake of simplicity, we assume that $\mu(\underline{\theta} \mid \underline{m}, t)=1$ for all $t \geq-1+\varepsilon$ to sustain the necessary off path play of Player $2 \underline{m} .{ }^{8}$

To show that this candidate can be made a full equilibrium, we will now construct a simultaneous-move global game $G^{\prime}$ based on the unsolved black hole part of the global signaling game. The equilibrium analysis of the game to be constructed might be of some interest in itself as it complements the analysis of Carlsson and van Damme (1993) in that it deals with the consequences of the global games approach in situations when only one mixed equilibrium exists for the unperturbed game.

There are two players, Player $1 \underline{\theta}$ ' and Player $2 \bar{m}$ '. Before the game, Nature determines the tuple $\left(\tilde{t}^{\prime}, t^{\prime}\right)$, where the joint distribution of $\left(t^{\prime}, \tilde{t}^{\prime}\right)$ is given by the conditional distribution $\nu^{\prime}$ that results when we impose the restriction

$$
\left(\tilde{t}^{\prime}, t^{\prime}\right) \in B \times B
$$

where

$$
B:=[0.8-2 \varepsilon, 1+2 \varepsilon] .
$$

\footnotetext{
${ }^{8}$ We have seen earlier that in any equilibrium Player $2 \underline{m}$ 's action has to be such that Player $1 \underline{\theta}$, given $\tilde{t} \in(-1,0.8)$, is deterred from sending message $\underline{m}$. The assumption $\mu(\underline{\theta} \mid \underline{m}, t)=1$ for $t \geq-1+\varepsilon$ guarantees that. It is satisfied anyway for all reached local information sets $(\underline{m}, t)$ of Player 2 and is therefore not restrictive.
} 
It is not difficult to see that this conditional distribution is given by the uniform distribution on the "thick diagonal"

$$
\left\{\left(\tilde{t}^{\prime}, t^{\prime}\right) \text { s.t. }\left|t^{\prime}-\tilde{t}^{\prime}\right| \leq \varepsilon\right\} .
$$

See Figure 5 for illustration. Strategies for Players $1 \underline{\theta}^{\prime}$ and $2 \bar{m}$ ' are given by - place functions

$$
\begin{aligned}
& \sigma_{1}^{\prime}: B \rightarrow \Delta(M), \\
& \sigma_{2}^{\prime}: B \rightarrow \Delta(R) .
\end{aligned}
$$

here -

The payoffs is this game are those accruing to the Players $1 \underline{\theta}$ and $2 \bar{m}$ in the global signaling game with the same signals $t$ and $\tilde{t}$, given that the strategies of Players $1 \bar{\theta}$ and $2 \underline{m}$ are as described in the candidate equilibrium. The payoff matrix of the game $G^{\prime}$ is depicted Figure 6 .

For the general case, it is shown in the appendix that the conditions for the existence theorem by Milgrom and Weber (1985) are satisfied, so that an equilibrium in $G^{\prime}$ exists. Moreover, as is also shown in the appendix, the equilibrium strategies in $G^{\prime}$ have to conform with the proposed incomplete equilibrium strategies of the global signaling game in the regions of overlap. Thus, we conclude:

Theorem 3. There exists an equilibrium in the global signaling game.

Proof. See the appendix.

The reader will notice that Theorem 3 establishes existence in mixed strategies only, which is sufficient for our purposes. While pure equilibria can be constructed in some examples, we have been unable to prove general existence. In particular, the Purification Theorem of Milgrom and Weber (1986) does not apply in our specific case, since types are not independent in global signaling games.

Together with Theorem 1, the above result implies that a Perfect Bayesian Equilibrium in the signaling game in question is informationally stable if and only if it is consistent with the Intuitive Criterion. Since the Intuitive Criterion is based on the ideas of stability expressed in Kohlberg and Mertens (1986), we expect that, similar to the results obtained by Carlsson and van Damme (1993), in the generalised case the global games argument is even more selective than the Intuitive Criterion. 


\section{The example (cont.)}

This section is entirely devoted to the example game for which we will complement the incomplete equilibrium strategies from the previous section for a specific range of $\varepsilon$ values. We hope that the concreteness of the arguments will further the understanding of the process going on behind the general more abstract analysis. This seems of special interest in the present context in which the impact of a global games approach to a game with a single mixed Nash equilibrium is studied. As will become clear from the analysis, there appears to be in fact little flexibility in defining equilibrium strategies even in the black hole area.

To start with, one should notice that the equilibrium just proved to exist must be one in which both of the players in the reduced game $G^{\prime}$, i.e. Player $1 \underline{\theta}$ and Player $2 \bar{m}$, at least seemingly mix their actions within the black hole area. The next argument will clarify what is meant by this.

We have seen earlier that, since in equilibrium Player $2 \underline{m}$ will play $\bar{r}$ whenever her information set is reached, it is optimal for Player $1 \underline{\theta}$ to play $\bar{m}$ if she assigns a probability larger than $1 / 2$ to Player $2 \bar{m}$ playing $\underline{r}$. On the other hand, we know that for types $t>t^{*}$ Player 2 prefers to play $\bar{r}$ if she assesses the probability of playing against Player $1 \underline{\theta}$ to be at least $1-p$, or even less as $t$ increases. Putting things together, it becomes intuitive that both players in equilibrium have to choose actions in such a way that the corresponding believes of their opponent always assign non-zero probability to both possibilities. We will now move on to the construction of one particular pair of equilibrium strategies; see Figure 7 for illustration.

It is instructive to consider the decision of Player $2 \bar{m}$ first. The aim is to identify the conditions under which Player $2 \bar{m}$ chooses either $\bar{r}$ or $\underline{r}$ (or is indifferent). Assume that Player $2 \bar{m}$ 's type is $t \in[0.8,1]$. Then, by definition of the information structure, Player $2 \bar{m}$ knows that Player $1 \underline{\theta}$ has received a signal $\tilde{t} \in[t-\varepsilon, t+\varepsilon]$. Remember that the probability with which Player $1 \underline{\theta}$ sends message $\underline{m}$ is given by $\sigma_{1}(\underline{m}, \underline{\theta}, \tilde{t})$. Thus, the probability Player $2 \bar{m}$ will assign to the event that a Player $1 \underline{\theta}$ of some type $\tilde{t} \in[t-\varepsilon, t+\varepsilon]$ sends the message $\underline{m}$ is given by

$$
\lambda(t):=\int_{t-\varepsilon}^{t+\varepsilon} \sigma_{1}(\underline{m}, \underline{\theta}, \tilde{t}) d \tilde{t} .
$$

Let $\beta(t):=\mu(\underline{\theta} \mid \bar{m}, t)$ denote the posterior probability that a Player $2 \bar{m}$ of 
type $t$ assigns to the global sender type $\theta=\underline{\theta}$. Then, by Bayes' rule,

$$
\begin{aligned}
\beta(t) & =\frac{\operatorname{Pr}(\theta=\underline{\theta} \text { and } m=\bar{m})}{\operatorname{Pr}(\theta=\underline{\theta} \text { and } m=\bar{m})+\operatorname{Pr}(\theta=\bar{\theta} \text { and } m=\bar{m})} \\
& =\frac{0.1 \cdot(1-\lambda(t))}{0.1 \cdot(1-\lambda(t))+0.9} \\
& =\frac{1-\lambda(t)}{10-\lambda(t)} .
\end{aligned}
$$

Consequently, Player $2 \bar{m}$ is indifferent if and only if

$$
(1+t) \cdot \beta(t)+(t-1) \cdot(1-\beta(t))=0 .
$$

Rearranging gives

$$
\beta(t)=\frac{1-t}{2} .
$$

Equating (2) and (3) yields

$$
\lambda^{*}(t)=10-\frac{18}{1+t} .
$$

This formula identifies the "indifference curve" for Player $2 \bar{m}$. It is depicted as a dotted convex-shaped curve reaching over the interval $[0.8,1]$ in the bottom part of Figure 7. The next lemma is an immediate consequence of the above discussion.

Lemma 2. Whenever $\lambda(t)<\lambda^{*}(t)$, Player $2 \bar{m}$ chooses $\bar{r}$; whenever $\lambda(t)>7$ $\lambda^{*}(t)$, Player $2 \bar{m}$ chooses $\underline{r}$.

Proof. See text above.

In the sequel, a solution will be offered where at the left boundary, i.e. for $t$ close to 0.8 , we have $\lambda(t)>\lambda^{*}(t)$, and at the right boundary, i.e. for $t$ close to 1 , we have $\lambda(t)<\lambda^{*}(t)$. Since beliefs $\lambda(t)$ must be continuous as a consequence of integrating out the noise in the signal to Player 1 , there will be a non-empty set of intersection points where $\lambda(t)=\lambda^{*}(t)$. For concreteness, we will focus on the case of exactly three intersection points

$$
0.8<t_{1}<t_{2}<t_{3}<1
$$

where

$$
t_{k}=t_{0}+k \varepsilon,
$$


and $t_{0}$ is a constant. Moreover, we assume that Player $2 \bar{m}$ 's strategy is a step function in the black hole. Specifically, Player $2 \bar{m}$ chooses $\underline{r}$ for signals $t<t_{1}$ and for $t \in\left(t_{2}, t_{3}\right)$; she chooses $\bar{r}$ for signals $t \in\left(t_{1}, t_{2}\right)$ and for $t>t_{3}$.

We now turn to Player $1 \underline{\theta}$. As her signal of Player 2's type is noisy, Player $1 \underline{\theta}$ 's belief is an integral over the actions chosen by Player $2 \bar{m}$. Given the assumptions made about Player $2 \bar{m}$, we get a piecewise linear belief (as depicted in Figure 7), that assigns probability smaller than $1 / 2$ to $\bar{r}$ for signals $\tilde{t}<t_{1}$, probability $1 / 2$ to $\bar{r}$ in the interval $\left[t_{1}, t_{3}\right]$, and probability larger than $1 / 2$ to $\bar{r}$ for values $\tilde{t}>t_{3}$. From the payoff structure, we deduce that Player $1 \underline{\theta}$ chooses $\bar{m}$ for signals $\tilde{t}<t_{1}$, and $\underline{m}$ for signals $\tilde{t}>t_{3}$. In the interval $\left[t_{1}, t_{3}\right]$, however, Player $1 \underline{\theta}^{\prime} \mathrm{s}$ strategy must be chosen in such a way that it induces the desired shape of the belief function $\lambda(t)$. Proving the proposition below requires the construction of a strategy for Player $1 \underline{\theta}$ that integrates into a belief $\lambda(t)$ for Player $2 \bar{m}$ such that the difference $\lambda(t)-\lambda^{*}(t)$ always has the desired sign. As will be shown in the appendix one possible strategy for Player $1 \underline{\theta}$ is to play $\underline{m}$ with probability

$$
\sigma_{1}(\underline{m}, \underline{\theta}, \tilde{t})=\frac{36 \varepsilon}{(1+\tilde{t}-\varepsilon)^{2}}
$$

for $\tilde{t} \in\left[t_{1}, t_{3}\right]$. This will provide the desired fixed point of the mapping starting at the top (or any other) segment of Figure 7, i.e. Player 2's strategy, and running through all the parts of the figure before returning to the strategy of Player 2 again.

Proposition 1. The strategy profile described above is an equilibrium in the global signaling game for $\varepsilon \in\left(\frac{1}{20}, \frac{1}{15}\right)$.

Proof. See the appendix.

The main conclusion from this Proposition is that even on the equilibrium path uniqueness of the global equilibrium cannot be guaranteed, at least not within the black hole. To see why, note that there is some freedom to specify the belief curve $\lambda(t)$. One could, for example, have $\lambda(t)$ closer to $\lambda^{*}(t)$ at the left boundary, levelling the difference out on the way to $t_{1}$.

As a further point note that in the constructed equilibrium only beliefs are close to those of the mixed equilibrium in the unperturbed game. This is because, as we have seen, players must not assign probability 1 to any pure action in the black hole in equilibrium. Thus, players' equilibrium choices must be perceived as mixing over any interval of length $2 \varepsilon$. This in turn 
implies that in any interval of length $2 \varepsilon$, the curve representing players' beliefs have to cross the indifference curve of the unperturbed game at least once. Continuity of beliefs and the frequency of strategy changes needed, therefore, explain why at least beliefs have to converge as $\varepsilon$ goes to zero.

\section{Conclusion}

In this paper, we have explored the consequences of applying the global games methodology to the theory of equilibrium selection in signaling games. It turned out that predictions of Cho and Kreps' standard refinement can be replicated for an economically relevant class of signaling games by introducing additional uncertainty, on the part of the sender, about the utility function of the receiver. We have argued that this specific assumption of additional uncertainty is in some circumstances easier to defend than alternative justifications. All in all, we hope to have convinced the reader that applying global games techniques also to signaling games is a valuable approach with potentially interesting economic applications.

\section{Appendix}

Proof of Theorem 1. The proof below follows the line of argument outlined in Section 1. By simple dominance, we obtain that for $t<-x$, Player $2 \bar{m}$ chooses action $\underline{r}$. Assume now that for some $t^{+}$, it has been shown that for any $t<t^{+}$, Player $2 \bar{m}$ replies with $\underline{r}$. Denote the probability Player 1 assigns to Player 2 responding to message $m$ with response $r$, conditional on her signal $\tilde{t}$, by $\operatorname{Pr}(r \mid m ; \tilde{t})$. Then Player $1 \bar{\theta}$ chooses $\bar{m}$ irrespective of her beliefs about $2 \underline{m}$ 's choice of action if

$$
\operatorname{Pr}(\bar{r} \mid \bar{m} ; \tilde{t}) \cdot d+(1-\operatorname{Pr}(\bar{r} \mid \bar{m} ; \tilde{t}))(b+d)>b .
$$

This is equivalent to $\operatorname{Pr}(\bar{r} \mid \bar{m} ; \tilde{t})<d / b$. However, since the noise is uniformly distributed, Player $1 \bar{\theta}$ will assign probability $\operatorname{Pr}(\bar{r} \mid \bar{m} ; \tilde{t})<d / b$ to the event that $2 \bar{m}$ chooses $\bar{r}$, for all

$$
\tilde{t}<t^{+}-\varepsilon\left(1-\frac{2 d}{b}\right)
$$

Thus, we have shown the following assertion. Assume that for all $t<t^{+}$, Player $2 \bar{m}$ responds with $\underline{r}$. Then for any $\tilde{t}$ satisfying (4), Player $1 \bar{\theta}$ chooses $\bar{m}$. Consider Player $2 \bar{m}$ again. Assume that Player $1 \bar{\theta}$ chooses message $\bar{m}$ for all values $\tilde{t}<t^{+}$for some value $t^{*}$. Consider the case in which Player 
$2 \bar{m}$ is of type $t<t^{+}+\varepsilon$. Given that $t^{\prime}$ is close to $t^{+}$, Player 2 knows that the probability of Player 1 receiving a signal $\tilde{t}<t^{+}$is strictly positive. By assumption, Player 1 chooses $\bar{m}$ for values $\tilde{t}<t^{+}$. Thus, Player 2's posterior on $\bar{m}$ assigns some strictly positive probability to $\theta=\bar{\theta}$. Moreover, given $\tilde{t}$, whenever $1 \underline{\theta}$ has a weak preference for $\bar{m}, 1 \bar{\theta}$ has a strict preference for $\bar{m}$ given the same signal $\tilde{t}$. Hence, the posterior of facing a global type $\bar{\theta}$ on message $\bar{m}$ cannot fall below the prior probability $p$ of the high type:

$$
\mu(\bar{\theta} \mid \bar{m}, t) \geq p
$$

Therefore, the expected payoff for $2 \bar{m}$, denoted by $\pi_{2}(. \mid \bar{m}, t)$, from $\bar{r}$ is bounded from above by

$$
\pi_{2}(\bar{r} \mid \bar{m}, t) \leq(1-p)(t+x)+p(t-y) .
$$

However, even this upper bound falls below the zero payoff from $\underline{r}$ whenever

$$
t<t^{*}:=p y-(1-p) x .
$$

So we have shown: Assume that $1 \bar{\theta}$ plays $\bar{m}$ for all $\tilde{t}<t^{+}<t^{*}-\varepsilon$. Then $2 \bar{m}$ chooses $\underline{r}$ for all $t<t^{*}+\varepsilon$. Putting the above facts together delivers the result that for any signal $\tilde{t}<t^{+}$, Player $1 \bar{\theta}$ chooses $\bar{m}$. Note also that, because of assumption $\mathrm{A}$, we have $t^{+}>0$, for sufficiently small $\varepsilon$. This proves that pooling on $\underline{m}$ is not an informationally stable equilibrium of the signaling game $G_{0}(0)$.

Next, we will consider Player 1 again. We know that $\bar{r}$ is strictly dominant for Player 2 if $t>y$. Expected payoffs $\pi_{1}(. \mid \underline{\theta}, \tilde{t})$ for Player $1 \underline{\theta}$ are given by

$$
\begin{aligned}
& \pi_{1}(\underline{m} \mid \underline{\theta}, \tilde{t})=\operatorname{Pr}(\bar{r} \mid \underline{m} ; \tilde{t}) \cdot a+\operatorname{Pr}(\underline{r} \mid \underline{m} ; \tilde{t}) \cdot(a+c) \\
& \pi_{1}(\bar{m} \mid \underline{\theta}, \tilde{t})=\operatorname{Pr}(\bar{r} \mid \bar{m} ; \tilde{t}) \cdot 0+\operatorname{Pr}(\underline{r} \mid \bar{m} ; \tilde{t}) \cdot c .
\end{aligned}
$$

Thus, message $\bar{m}$ is optimal for Player $1 \underline{\theta}$ if

$$
\frac{a}{c}+\operatorname{Pr}(\underline{r} \mid \underline{m} ; \tilde{t})<\operatorname{Pr}(\underline{r} \mid \bar{m} ; \tilde{t}) .
$$

Player $1 \underline{\theta}$ plays $\bar{m}$ if she assigns at least probability $a / c$ to the event that Player $2 \bar{m}$ will respond with $\underline{r}$. To show now that in equilibrium also $1 \underline{\theta}$ has to choose $\bar{m}$ for $\tilde{t}=0$, there are two things to check. First of all, we need that $t^{*}$ is larger than zero, which, as noted earlier, is equivalent to requiring

$$
p>\frac{x}{x+y} .
$$


This ensures that, at least for small values of $\varepsilon$, the high type of Player 1 send $\bar{m}$ in a $2 \varepsilon$-neighbourhood of $\tilde{t}=0$ and that indeed Player $2 \bar{m}$ will respond with $\underline{r}$ for types $t \in U_{2 \varepsilon}:=[-2 \varepsilon, 2 \varepsilon]$. Now, given this, assume as in the example that there is an equilibrium in which $1 \underline{\theta}$ sends $\underline{m}$ in a set $\mathrm{U}$ of strictly positive measure, where $\mathrm{U}$ is contained in an $\varepsilon^{*}$-neighbourhood of zero, with

$$
\varepsilon^{*} \leq \frac{1}{2}\left(1-\frac{a}{c}\right) \cdot \varepsilon .
$$

Next consider Player 2 given type $t \in U_{2 \varepsilon}$ and observing $\underline{m}$. In equilibrium Player 2 has to assign positive posterior probability to $\underline{m}$. Using Fact 3.6, Player 2 has to believe with probability 1 that this action was chosen by a Player 1 of global type $\underline{\theta}$. Thus, Player $2 \underline{m}$ has to respond with $\bar{r}$ with probability 1 for all types $t \in\left[-\left(\varepsilon+\varepsilon^{*}\right), \varepsilon+\varepsilon^{*}\right]$. This in turn leads Player 1 to believe that given $\tilde{t} \in U$

$$
\operatorname{Pr}(\bar{r} \mid \underline{m} ; \tilde{t})>\frac{a}{c}
$$

Therefore, having the above optimality condition in mind, we can conclude that $1 \underline{\theta}$ sends message $\bar{m}$. This proves: In any equilibrium, the global type $\underline{\theta}$ of Player 1 sends $\bar{m}$ in a small neighbourhood of $\tilde{t}=0$, given $\varepsilon$ is sufficiently small. Moreover the beliefs of $1 \underline{\theta}$ have to be such that she assigns a probability larger than or equal to a/c to Player $2 \underline{m}$ responding with $r$. This completes the proof.

Proof of Theorem 2. To obtain the general result, note that, due to the payoff structure, the main special feature of the example game $G(\varepsilon)$ is that especially for Player $1 \underline{\theta}$ (but also for Player $1 \bar{\theta}$ ) cut off probabilities always are at $50 \%$. Consequently, whenever Player 2's behaviour is specified up to some type $t^{+}$Player $1 \underline{\theta}^{\prime} s$ cut off signal $\tilde{t}^{+}$will be equal to $t^{+}$since the most extreme that can happen is a complete change in behaviour (leading to a 50/50 assessment). However, as we have seen in the general proof of Theorem 1, it may suffice for Player $1 \underline{\theta}$ to expect a smaller (or larger) share of types of Player 2 to play a different action in the general case. Specifically, this share was shown to be given by the fraction $a / c$. Moreover, the single crossing property used for the argument in the specific example still exists in the general case. Thus, if we define switching points accordingly, i.e. if we define: 


$$
\begin{aligned}
t^{*} & :=p y-(1-p) x, \\
x^{\circ} & :=x+\varepsilon\left(1-\frac{2 a}{c}\right), \\
y^{\circ} & :=y+\varepsilon\left(1-\frac{2 a}{c}\right), \\
t^{\circ} & :=t^{*}+\varepsilon\left(1-\frac{2 a}{c}\right),
\end{aligned}
$$

and follow exactly the same lines of argument as in Section 4, we get that in any equilibrium of the generalised game we must have:

- $1 \bar{\theta}$ plays $\bar{m}$ irrespective of her signal;

- $1 \underline{\theta}$ plays $\underline{m}$ for $\tilde{t} \leq-x^{\circ}$ and $\tilde{t}>y^{\circ}$, and message $\bar{m}$ for $\tilde{t} \in\left(-x^{\circ}, t^{\circ}\right)$;

- $2 \bar{m}$ plays $\underline{r}$ for $t<t^{*}$, and $\bar{r}$ for $t>y$;

- $2 \underline{m}$ plays $\underline{r}$ for $t<-x$ and $\bar{r}$ for $t>y$ and for all types $t \in\left[-x, t^{*}\right]$ plays such that Player $1 \underline{\theta}$, given a signal $\tilde{t} \in\left(-x^{\circ}, t^{\circ}\right)$, is deterred from sending message $\underline{m}$.

Obviously, for the example game $G(\varepsilon)$, we get $t^{*}=0.8, x^{\circ}=1, y^{\circ}=1$, and $t^{\circ}=t^{*}=0.8$ corresponding to our earlier results.

Proof of Theorem 3. The general proof has three steps, the outline of which reads as follows. We start by making the same kind of educated guess about the equilibrium strategies outside the black hole as for the example game and again reduce the game to a two-by-two normal form game which on its own - as we show - is amenable to the Milgrom/Weber results. Secondly, we show that the suggested strategies must actually conform with the Milgrom/Weber solution for the reduced game in the the regions of overlap. Finally, we prove that the resulting strategies for the global signaling game constitute a perfect Bayesian Equilibrium.

Step 1. Since, as noted earlier, in the general case switching points are different, we again use the constants $t^{*}, x^{\circ}, y^{\circ}$ and $t^{\circ}$ as defined in the generalised proof of Theorem 2. Next, we propose the incomplete candidate for an equilibrium strategy based on the findings in the earlier general uniqueness discussion (cf. Figure 4):

- Player $1 \bar{\theta}$ chooses $\bar{m}$ throughout. 
- Player $1 \underline{\theta}$ chooses message $\underline{m}$ for $\tilde{t} \leq-x^{\circ}$, message $\bar{m}$ for $\tilde{t} \in\left(-x^{\circ}, t^{\circ}\right)$, and message $\underline{m}$ for $\tilde{t}>y^{\circ}$.

- Player $2 \bar{m}$ chooses $\underline{r}$ for $t<t^{*}$, and $\bar{r}$ for $t>y$.

- Player $2 \underline{m}$ chooses $\underline{r}$ for $t<-x$, and $\bar{r}$ for $t \geq-x$.

This specification fully describes choices of actions for Players $1 \bar{\theta}$ and $2 \underline{m}$. Moreover, it describes choices of action for Players $1 \underline{\theta}$ for signals $\tilde{t} \notin\left[t^{\circ}, y^{\circ}\right]$ and for Player $2 \bar{m}$ for types $t \notin\left[t^{*}, y\right]$. Also, we again assume that $\mu(\underline{\theta} \mid \underline{m}, t)=$ 1 for all $t \geq x^{\circ}+\varepsilon$ to guarantee the necessary off path play of Player $2 \underline{m} .{ }^{9}$

The corresponding simultaneous-move Bayesian game $G^{\prime}$ between Player $1 \underline{\theta}^{\prime}$ and Player $2 \bar{m}$ ' follows the same steps as in the example. First, Nature determines the tuple $\left(\tilde{t}^{\prime}, t^{\prime}\right)$ according to the conditional distribution $\nu^{\prime}$ that results when we impose the restriction

$$
\left(\tilde{t}^{\prime}, t^{\prime}\right) \in B \times B
$$

where

$$
B:=\left[t^{*}-2 \varepsilon, y+2 \varepsilon\right] .
$$

The conditional distribution again is uniform on the "thick diagonal"

$$
\left\{\left(\tilde{t}^{\prime}, t^{\prime}\right) \text { s.t. }\left|t^{\prime}-\tilde{t}^{\prime}\right| \leq \varepsilon\right\} .
$$

Strategies for Players 1' and $2 \bar{m}$ ' are given by

$$
\begin{aligned}
\sigma_{1}^{\prime} & : B \rightarrow \Delta(M), \\
\sigma_{2}^{\prime} & : B \rightarrow \Delta(A) .
\end{aligned}
$$

The payoffs of this game are those accruing to the Players 1 and $2 \bar{m}$ in the global signaling game with the same signals $t$ and $\tilde{t}$, given that the strategies of Players $1 \bar{\theta}$ and $2 \underline{m}$ are as described in the candidate equilibrium.

Lemma A1. The constructed two-by-two game $G^{\prime}$ satisfies the requirements of the Milgrom Weber theorem. Thus, there exists an equilibrium of $G^{\prime}$.

Proof. To apply the general existence theorem of Milgrom and Weber (1985), we have to check for equicontinuous payoffs and absolute continuous information. The former is satisfied since all action spaces are finite. The latter holds since, as $\varepsilon>0$, the conditional distribution $\nu^{\prime}$ is absolute

\footnotetext{
${ }^{9}$ As noted earlier, this assumption is not restrictive.
} 
continuous with respect to the product uniform distribution on $B \times B$, as any null set in $B \times B$ of the latter clearly has measure 0 also under $\nu^{\prime}$. Hence, there exists an equilibrium in distributional strategies in $G^{\prime}$.

The next step is to show that we can use the equilibrium in $G^{\prime}$ to construct an equilibrium in the global signaling game. We specify strategies as follows: Players $1 \bar{\theta}$ and $2 \bar{m}$ choose the strategies described in the candidate equilibrium; Players $1 \underline{\theta}$ and $2 \underline{m}$, however, choose the actions identified in the candidate profile only for signals and types outside the intervals $\left[t^{\circ}, y^{\circ}\right]$ and $\left[t^{*}, y\right]$, respectively; Player $1 \underline{\theta}$, for signals $\tilde{t} \in\left[t^{\circ}, y^{\circ}\right]$, and Player $2 \underline{m}$, for $t \in\left[t^{*}, y\right]$, choose the (mixed) actions specified by the Milgrom/Weber equilibrium.

Step 2. We will first check that the Milgrom/Weber equilibrium conforms with the candidate strategies in the regions of overlap. Refer to Figures 3 and 5 for illustration. Consider first Player $2 \bar{m}$. We have to show that the Milgrom/Weber equilibrium involves playing $\underline{r}$ for $t \in\left[t^{*}-2 \varepsilon, t^{*}\right)$, and playing $\bar{r}$ for $t \in(y, y+2 \varepsilon]$. Assume first $t \in\left[t^{*}-2 \varepsilon, t^{*}\right)$. Since Player $1 \bar{\theta}$ chooses $\bar{m}$ throughout, a payoff comparison shows that Player $2 \bar{m}$ ' will necessarily choose $\underline{r}$ whenever

$$
(1-p)(t+x)+p(t-y)>0 .
$$

This, however, is equivalent to $t<t^{*}$. Thus, the Milgrom/Weber equilibrium involves playing $\underline{r}$ for all $t \in\left[t^{*}-2 \varepsilon, t^{*}\right)$. Assume now that $t \in(y, y+2 \varepsilon]$. Since action $\bar{r}$ is dominant for Player $2 \bar{m}$, for $t>y$, the Milgrom/Weber equilibrium involves playing $\bar{r}$ for $t \in(y, y+2 \varepsilon]$.

Next, consider Player $1 \underline{\theta}$. A payoff comparison shows that Player 1' necessarily chooses message $\bar{m}$ if

$$
\operatorname{Pr}(\underline{r} \mid \bar{m} ; \tilde{t}) \cdot c>a .
$$

This is certainly satisfied for all $\tilde{t} \in\left[t^{*}-2 \varepsilon, t^{*}-\varepsilon\right]$, because for those signals, as shown above, $\operatorname{Pr}(\underline{r} \mid \bar{m} ; \tilde{t})=1$. For the remaining values of $\tilde{t} \in\left(t^{*}-\varepsilon, t^{\circ}\right)$, a short calculation shows that (5) is necessarily satisfied whenever $\tilde{t}<t^{\circ}$, by definition of $t^{\circ}$. Consider now the case $\tilde{t} \in\left(y^{\circ}, y+2 \varepsilon\right]$. Player $1^{\prime}$ necessarily chooses message $\underline{m}$ if

$$
a>\operatorname{Pr}(\underline{r} \mid \bar{m} ; \tilde{t}) \cdot c .
$$

This is certainly the case when $\tilde{t} \in[y+\varepsilon, y+2 \varepsilon]$, because due to dominance it must hold $\operatorname{Pr}(\bar{r} \mid \bar{m} ; \tilde{t})=1$. For $\tilde{t} \in\left(y^{\circ}, y+\varepsilon\right)$, the optimality of $\underline{m}$ follows again from a short calculation. This shows that the Milgrom/Weber equilibrium and the candidate strategies conform in the intervals of overlap. 
Step 3. Next, we prove that the strategy profile constructed in Section 4 indeed constitutes a Perfect Bayesian Equilibrium. We start with Player $1 \bar{\theta}$. Whatever the signal $\tilde{t}$ of Player $1 \bar{\theta}$, it must either hold that Player $\operatorname{Pr}(\underline{r} \mid \bar{m} ; \tilde{t})=1$ (for low values of $\tilde{t}$ ) or that $\operatorname{Pr}(\bar{r} \mid \underline{m} ; \tilde{t})=1$ (for high values of $\tilde{t})$, or both. In either case, message $\bar{m}$ is the optimal choice for $1 \bar{\theta}$.

To move on, consider Player $2 \bar{m}$. Given that $1 \bar{\theta}$ plays $\bar{m}$ throughout, any information set for Player $2 \bar{m}$ is reached, and it must always hold that $\mu(\bar{\theta} \mid \bar{m}, t) \geq p$. A payoff comparison shows then that Player $2 \bar{m}$ will necessarily choose $\underline{r}$ when

$$
(1-p)(t+x)+p(t-y)>0 .
$$

This condition, however, is equivalent to $t<t^{*}$. On the other hand, for $t>y$, action $\bar{r}$ is dominant for Player $2 \bar{m}$. We can therefore restrict attention to values $t \in\left[t^{*}, y\right]$. To show that the action prescribed by $\sigma_{2}^{\prime}$ is optimal, it suffices to prove that $\sigma_{1}^{\prime}$ conforms with the candidate strategy for Player $1 \underline{\theta}$ in the intervals of overlap $\left[t^{*}-2 \varepsilon, t^{*}\right)$ and $[y, y+2 \varepsilon]$. But this has been done is step 2 of the proof.

Next, consider Player $1 \underline{\theta}$. For low values of $\tilde{t}$, i.e., for $\tilde{t}<t^{*}-\varepsilon$, we have $\operatorname{Pr}(\underline{r} \mid \bar{m} ; \tilde{t})=1$. In this case, message $\underline{m}$ is optimal if and only if $\tilde{t} \leq-x^{\circ}$, which is consistent with the candidate strategy. For very high values of $\tilde{t}$, i.e. for $\tilde{t}>y+\varepsilon$, we have $\operatorname{Pr}(\bar{r} \mid \bar{m} ; \tilde{t})=\operatorname{Pr}(\bar{r} \mid \underline{m} ; \tilde{t})=1$. In this case, message $\underline{m}$ is optimal for $1 \underline{\theta}$. So we can restrict attention to the interval $\left[t^{*}-\varepsilon, y+\varepsilon\right]$. However, for these values the optimality of Player $1 \underline{\theta}$ 's strategy follows from the optimality property of the strategy of Player $1 \underline{\theta}$ in the Milgrom/Weber equilibrium.

Finally, consider Player $2 \underline{m}$. A short calculation using the definition of $x^{\circ}$ shows that the candidate strategy for Player $2 \underline{m}$ is optimal. This completes the proof of Theorem 3 .

Proof of Proposition 1. We have to construct a strategy $\rho(s):=\sigma_{1}(\underline{m}, \underline{\theta}, s)$ 
for Player $1 \underline{\theta}$ that satisfies the following four sign conditions

$$
\begin{aligned}
& \frac{1}{2 \varepsilon} \int_{t-\varepsilon}^{t+\varepsilon} \rho(s) d s \geq \lambda^{*}(t) \quad \text { for } \quad t \leq t_{0}+\varepsilon \\
& \frac{1}{2 \varepsilon} \int_{t-\varepsilon}^{t+\varepsilon} \rho(s) d s \leq \lambda^{*}(t) \text { for } t \in\left[t_{0}+\varepsilon, t_{0}+2 \varepsilon\right] \\
& \frac{1}{2 \varepsilon} \int_{t-\varepsilon}^{t+\varepsilon} \rho(s) d s \geq \lambda^{*}(t) \text { for } t \in\left[t_{0}+2 \varepsilon, t_{0}+3 \varepsilon\right] \\
& \frac{1}{2 \varepsilon} \int_{t-\varepsilon}^{t+\varepsilon} \rho(s) d s \leq \lambda^{*}(t) \text { for } t \in\left[t_{0}+3 \varepsilon, t_{0}+4 \varepsilon\right] .
\end{aligned}
$$

In the sequel, we will re-formulate these conditions. Write

$$
h_{k}:=\lambda^{*}\left(t_{0}+k \cdot \varepsilon\right) .
$$

Since $\rho(s)=0$ for $s<t_{0}+\varepsilon$, we may rewrite (6) as

$$
\int_{t_{0}+\varepsilon}^{t} \rho(s) d s \geq 2 \varepsilon \cdot \lambda^{*}(t-\varepsilon) \text { for } t \in\left[t_{0}+\varepsilon, t_{0}+2 \varepsilon\right] .
$$

We know that we need to have

$$
\frac{1}{2 \varepsilon} \int_{t_{0}+\varepsilon}^{t_{0}+3 \varepsilon} \rho(s) d s=h_{2}
$$

and that $\rho(s)=1$ for $s \geq t_{0}+3 \varepsilon$. Hence, condition (8) is equivalent to

$$
\begin{aligned}
& \frac{1}{2 \varepsilon} \int_{t_{0}+\varepsilon}^{t-\varepsilon} \rho(s) d s \\
& =\frac{1}{2 \varepsilon}\left\{\int_{t_{0}+\varepsilon}^{t_{0}+3 \varepsilon} \rho(s) d s+\int_{t_{0}+3 \varepsilon}^{t+\varepsilon} \rho(s) d s-\int_{t-\varepsilon}^{t+\varepsilon} \rho(s) d s\right\} \\
& \leq h_{2}+\frac{t+\varepsilon-\left(t_{0}+3 \varepsilon\right)}{2 \varepsilon}-\lambda^{*}(t)
\end{aligned}
$$

for $t \in\left[t_{0}+2 \varepsilon, t_{0}+3 \varepsilon\right]$. Replacing $t$ by $t+\varepsilon$,we obtain

$$
\int_{t_{0}+\varepsilon}^{t} \rho(s) d s \leq 2 \varepsilon \cdot h_{2}+\left(t-t_{0}-\varepsilon\right)-2 \varepsilon \cdot \lambda^{*}(t+\varepsilon)
$$

for $t \in\left[t_{0}+\varepsilon, t_{0}+2 \varepsilon\right]$.

Now, putting the reformulated conditions together, we can give an upper and a lower bound for the integral of $\rho(s)$ for $t \in\left[t_{0}+\varepsilon, t_{0}+2 \varepsilon\right]$ :

$$
2 \varepsilon \cdot \lambda^{*}(t-\varepsilon) \leq \int_{t_{0}+\varepsilon}^{t} \rho(s) d s \leq 2 \varepsilon \cdot h_{2}+\left(t-t_{0}-\varepsilon\right)-2 \varepsilon \cdot \lambda^{*}(t+\varepsilon) .
$$


Similarly, using (7) and (9) we find the following condition for values of $\mathrm{t}$ in $\left[t_{0}+2 \varepsilon, t_{0}+3 \varepsilon\right]:$

$$
2 \varepsilon \cdot h_{3}+\left(t-t_{0}-2 \varepsilon\right)-2 \varepsilon \cdot \lambda^{*}(t+\varepsilon) \leq \int_{t_{0}+2 \varepsilon}^{t} \rho(s) d s \leq 2 \varepsilon \cdot \lambda^{*}(t-\varepsilon)-\varepsilon \cdot 2 h_{1} .
$$

The next step before we can construct an action $\rho(s)$ is to check wether both A and B are consistent. Starting with condition A, straightforward computations - beginning with the corner points and then showing that the result extends to the whole interval - show that this condition is satisfied for $\varepsilon$ - values satisfying $1 / 18 \leq \varepsilon \leq 9 / 100$. Condition $\mathrm{B}$ can be shown to be generally satisfied by checking the corner points first and then using the concavity of $\lambda^{*}$ and the result stated in Lemma A2 below.

Now we can construct an action profile $\rho(s)$ for Player $1 \underline{\theta}$. In the interval $\left[t_{0}+\varepsilon, t_{0}+2 \varepsilon\right]$ we simply take the lower bound of the constraint and try to stick to it, i.e. try to stay on $\lambda^{*}($.$) . Thus we get$

$$
2 \varepsilon \cdot \lambda^{*}(t-\varepsilon)=\int_{t_{0}+\varepsilon}^{t} \rho(s) d s
$$

Taking derivatives gives

$$
2 \varepsilon \cdot \frac{\partial \lambda^{*}}{\partial t}(t-\varepsilon)=\rho(t)
$$

Proceeding in the same way with the upper bound on the left-hand side of condition $\mathrm{B}$ on the interval $\left[t_{0}+\varepsilon, t_{0}+3 \varepsilon\right]$, we obtain

$$
\rho(\tilde{t})= \begin{cases}0 & \text { for } \tilde{t} \leq t_{0}+\varepsilon \\ \frac{36 \varepsilon}{(1+\tilde{t}-\varepsilon)^{2}} & \text { for } \tilde{t} \in\left[t_{0}+\varepsilon, t_{0}+3 \varepsilon\right] \\ 1 & \text { for } \tilde{t} \geq t_{0}+2 \varepsilon\end{cases}
$$

It only remains to check that $\rho(\tilde{t})<1$, i.e. if

$$
\frac{36 \varepsilon}{(1+\tilde{t}-\varepsilon)^{2}} \leq 1
$$

If we use that $(1+\tilde{t}-\varepsilon)^{2}>(1.8)^{2}$ we obtain:

$$
\frac{36}{1.8^{2}} \varepsilon=\frac{20}{1.8} \varepsilon=\frac{10}{0.9} \varepsilon \leq 1
$$

This holds since we know that $\varepsilon \leq 0.9 / 10$ by assumption. 
Lemma A2. The parameter $t_{0}$ is implicitly characterized by the equation

$$
\rho^{*}\left(t_{1}\right)-\rho^{*}\left(t_{2}\right)+\rho^{*}\left(t_{3}\right)=\frac{1}{2} .
$$

Proof of Lemma A2. This is almost an immediate consequence of the definition of $\rho^{*}($.$) . Write \rho(s):=\sigma_{1}(\underline{m}, \underline{\theta}, s)$. Then

$$
\begin{aligned}
& \rho^{*}\left(t_{1}\right)-\rho^{*}\left(t_{2}\right)+\rho^{*}\left(t_{3}\right) \\
& =\frac{1}{2 \varepsilon}\left\{\int_{t_{0}}^{t_{2}} \rho(s) d s-\int_{t_{1}}^{t_{3}} \rho(s) d s+\int_{t_{2}}^{t_{4}} \rho(s) d s\right\} \\
& =\frac{1}{2 \varepsilon}\left\{\int_{t_{0}}^{t_{1}} \rho(s) d s+\int_{t_{3}}^{t_{4}} \rho(s) d s\right\}=\frac{1}{2} .
\end{aligned}
$$

This proves the Lemma. 


\section{References}

Aumann, R. J., 1964, "Mixed and Behavior Strategies in Infinite Extensive Games," Advances in Game Theory, Ann. of Math. Studies No. 52, Princeton University Press, Princeton, N.J., 627-650.

Battigalli, P. and M. Siniscalchi, 2002, "Strong Belief and Forward Induction Reasoning," Journal of Economic Theory 106, pp. 356-391.

Banks, J. and J. Sobel, 1987, "Equilibrium Selection in Signalling Games," Econometrica 55, pp. 647-662.

Carlsson, H. and E. van Damme, 1993, "Global Games and Equilibrium Selection," Econometrica 61, pp. 989-1018.

Carlsson, H., and S. Dasgupta, 1997, "Noise-Proof Equilibria in Two-Action Signaling Games, Journal of Economic Theory 77, pp. 432-460.

Cho, I.-K. and D. M. Kreps, 1987, "Signalling Games and Stable Equilibria," Quarterly Journal of Economics 102, pp. 179-221.

Fudenberg, D. and J. Tirole, 1991a, "Perfect Bayesian Equilibrium and Sequential Equilibrium," Journal of Economic Theory 53, pp. 236-260.

Fudenberg, D. and J. Tirole, 1991b, Game Theory, MIT Press, Cambridge, Massachusetts.

Kohlberg, E., and Mertens, J. F., 1986, "On the Strategic Stability of Equilibria," Econometrica 54, pp. 1003-1037.

Milgrom, P. and R. Weber, 1985, "Distributional Strategies for Games with Incomplete Information," Mathematics of Operations Research 10, pp. 619631.

Morris, S. and H. S. Shin, 1998, "Unique Equilibrium in a Model of SelfFulfilling Currency Attacks," American Economic Review 88, pp. 587-597.

Morris, S. and H. S. Shin, 2003, "Global Games: Theory and Applications," in: Dewatripont, M., L. Hansen, and S. Turnovsky (eds.), Advances in Economics and Econometrics, the Eighth World Congress, Cambridge, UK, in press.

Spence, M. 1973, "Job Market Signaling," Quarterly Journal of Economics 87, pp. 355-74.

van Damme, E., 1991, Stability and Perfection of Nash Equilibria, Springer, 2nd edition. 


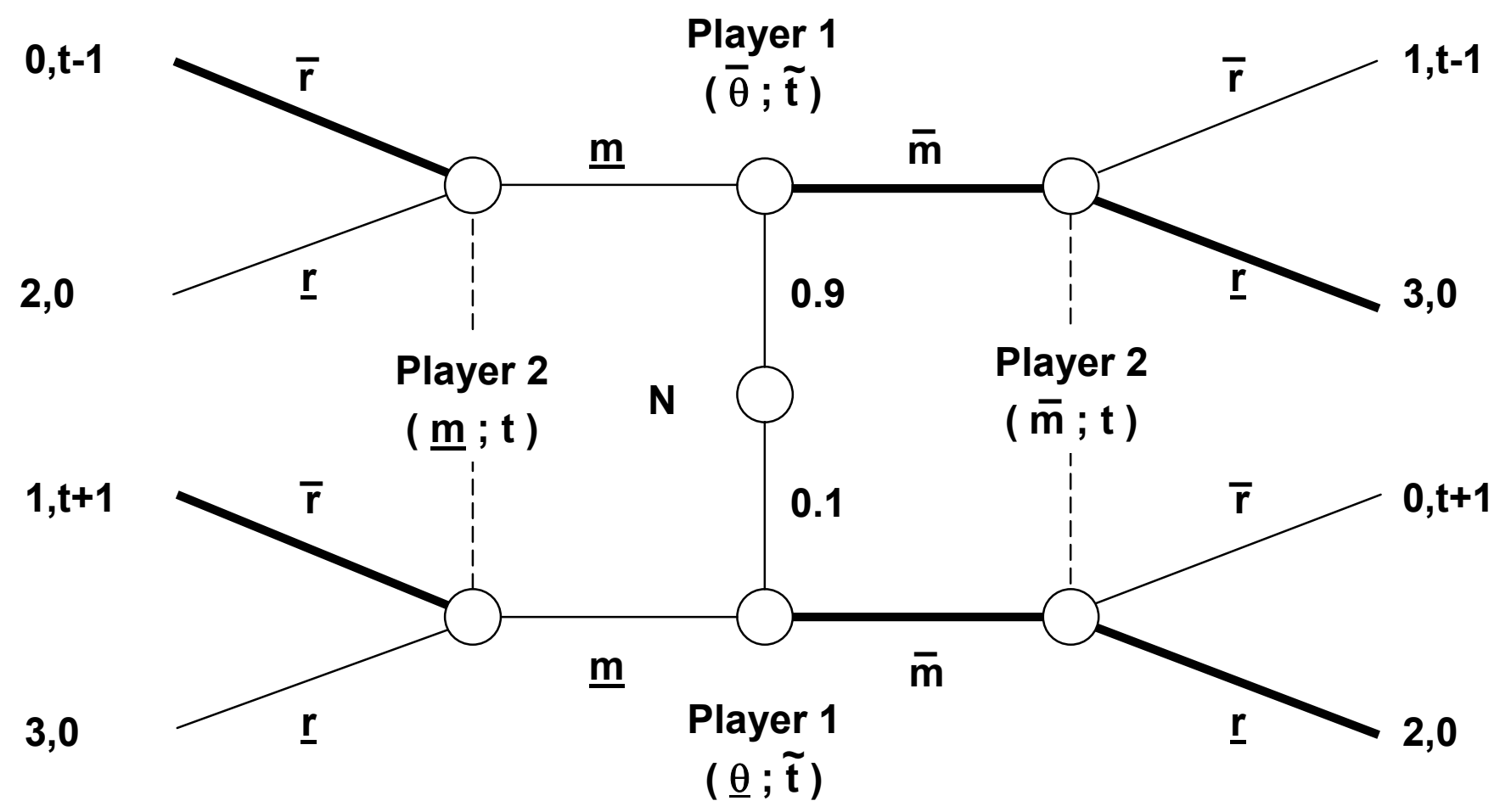

Figure 1 


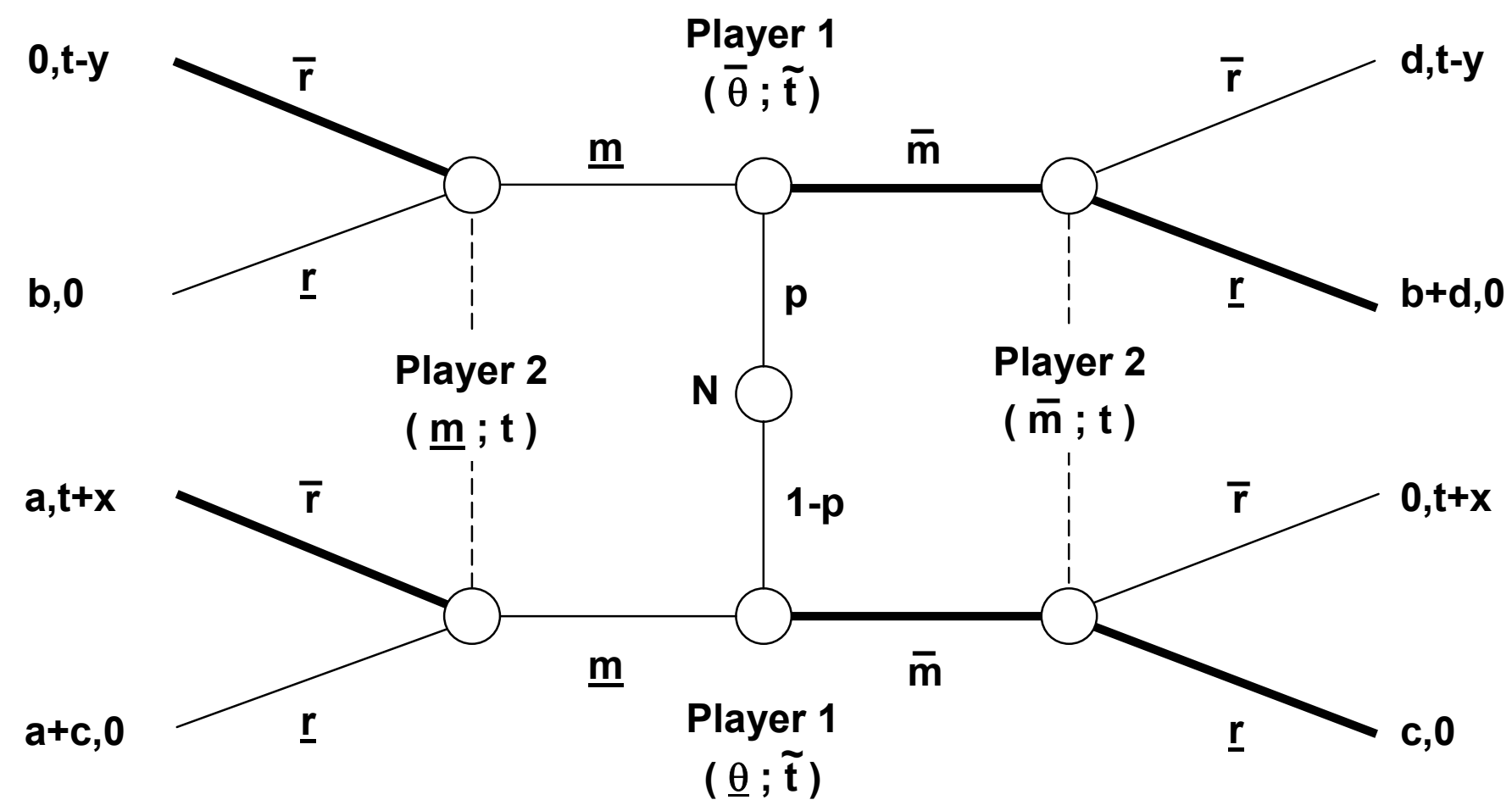

Figure 3 


\begin{tabular}{|c|c|c|}
\hline & 0 & \\
\hline message $\underline{\mathrm{m}}$ & message $\overline{\mathrm{m}}$ & message $\underline{\mathrm{m}}$ \\
\hline
\end{tabular}

er 2

action $\underline{r}$

Figure 4 


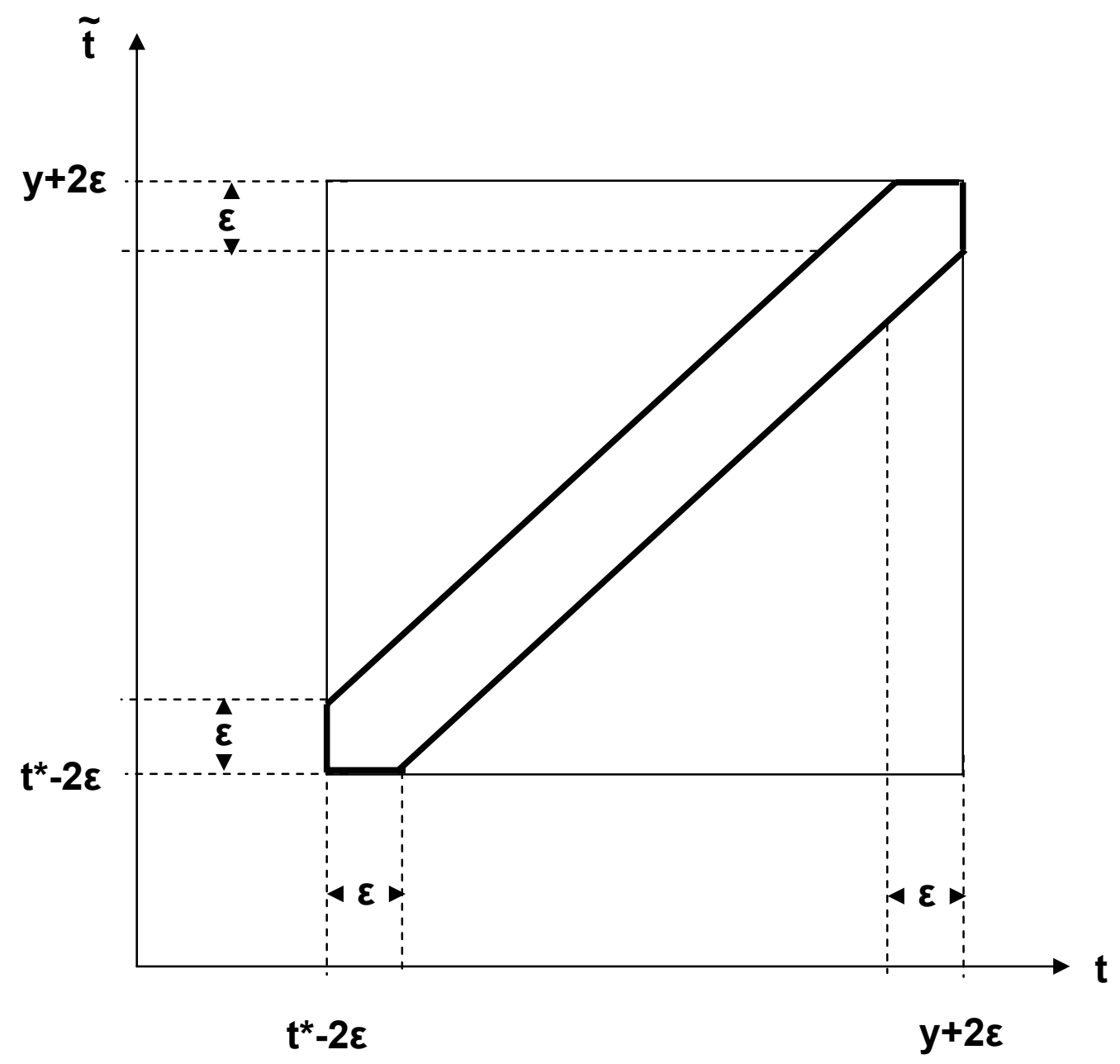

Figure 5 
Player $2 \overline{\mathbf{m}}^{\prime}$

G':

Player $1 \underline{\theta}^{\prime}$

\begin{tabular}{|c|c|}
\multicolumn{1}{c|}{$\underline{r}$} & $\bar{r}$ \\
\hline 1,0 & $1, \mathrm{t}-1$ \\
\hline 2,0 & $0, \mathrm{t}-0.8$ \\
\hline
\end{tabular}

Figure 6 


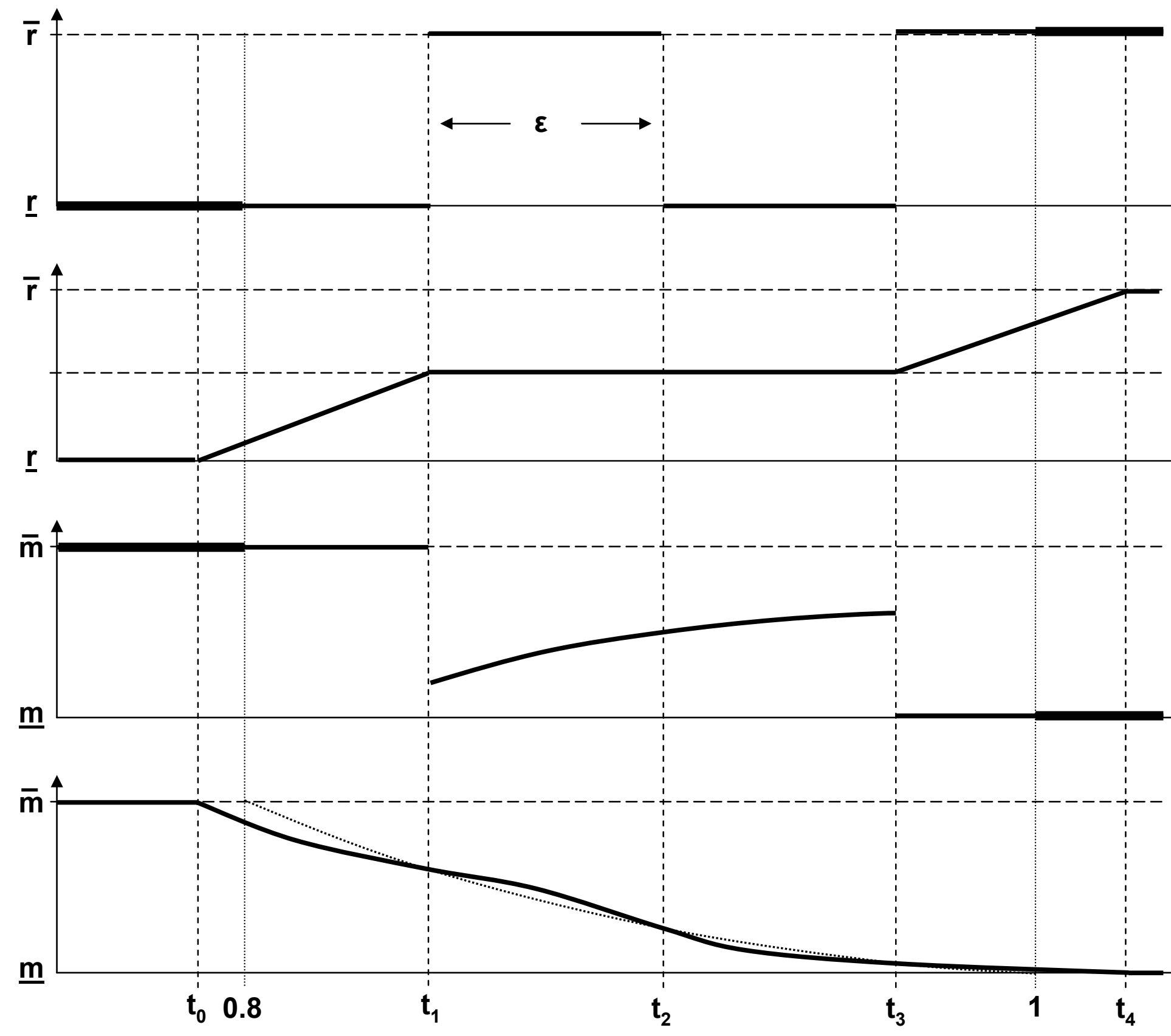

